\title{
Bem-estar em pessoas idosas institucionalizadas durante a pandemia: Uma revisão integrativa
}

\author{
Helga Rafael Henriques', Tiago Nascimento ${ }^{1}$, Andreia Costa ${ }^{1}$, \\ Cândida Durão', Mara Pereira Guerreiro ${ }^{1}$ e Cristina Baixinho²
}

1 eduCOVID Project | Escola Superior de Enfermagem de Lisboa; Centro de Investigação, Inovação e Desenvolvimento em Enfermagem de Lisboa (CIDNUR), Lisboa, Portugal | hrafael@esel.pt; tnascimento@esel.pt; andreia.costa@esel.pt; candida.durao@esel.pt; mara.guerreiro@esel.pt | https://orcid.org/0000-0003-2946-4485; https://orcid.org/00000003-3646-9057; https://orcid.org/0000-0002-2727-4402; https://orcid.org/0000-0002-9465528; https://orcid.org/0000-0001-8192-6080

${ }^{2}$ eduCOVID Project | Escola Superior de Enfermagem de Lisboa, Centro de Investigação, Inovação e Desenvolvimento em Enfermagem de Lisboa (CIDNUR), Lisboa, Portugal ciTechaCare, Leiria, Portugal | crbaixinho@esel.pt | https://orcid.org/0000-0001-7417-1732

Resumo: Introdução. As pessoas idosas residentes em Estruturas Residenciais para Pessoas Idosas (ERPI) são um dos grupos de maior vulnerabilidade na pandemia por COVID19. As medidas de distanciamento social inerentes à prevenção da COVID-19 poderão influenciar o bem-estar das pessoas institucionalizadas. Objetivos. Identificar estudos que permitam compreender o bem-estar das pessoas idosas residentes em ERPI face ao cumprimento das medidas de prevenção e controlo de infeção para a COVID-19; Métodos. Revisão integrativa da literatura de acordo com a estratégia PCC (população, conceito e contexto) em que $\mathrm{P}$ corresponde às Pessoas idosas sujeitas a medidas de prevenção e controlo de infeção para a COVID-19, C ao Bem-estar e C às ERPI. Foram consultadas as bases de dados MEDLINE, CINAHL, Pubmed, SCOPUS e Web of Science. A colheita de dados decorreu em março de 2021. A pesquisa e seleção dos artigos foram orientadas pela Preferred Reporting Items for Transparent Reporting of Systematic Reviews and MetaAnalyses. A avaliação da qualidade seguiu a Joanna Briggs International. Resultados. Foram identificados seis artigos que avaliaram o bem-estar de pessoas idosas institucionalizadas durante a pandemia. Os resultados sugerem que as medidas de prevenção e controle de infeção implementadas em ERPI poderão ter efeitos no bem-estar percecionado pelas pessoas idosas residentes, pela perda de contacto humano e pela perda de espaço de circulação, com declínio cognitivo, perda de capacidade funcional, mal-estar emocional e solidão. Conclusões. As medidas de prevenção e controlo implementadas nas ERPI no contexto da pandemia por COVID-19 parecem ter influenciado negativamente o bem-estar das pessoas idosas residentes.

Palavras-chave: Lares; Envelhecimento; Bem-Estar; COVID-19; Controle de Infeção.

Well-Being in Institutionalized Aging People During the Pandemic: An Integrative Review

Abstract: Introduction. Aging people living in nursing homes are one of the most vulnerable groups in the COVID-19 pandemic. The measures for COVID-19 prevention may influence the well-being of institutionalized people. Goals. To identify studies that allow us to understand the well-being of aging people living in nursing homes given compliance with the infection prevention and control measures for COVID-19. Methods. Integrative literature review according to the PCC strategy (population, concept, and context) in which P corresponds to aging people under the infection prevention and control measures for COVID-19, C is Wellbeing and $\mathrm{C}$ is nursing homes. We consulted MEDLINE, CINAHL, Pubmed, SCOPUS, and Web of Science databases. Data collection took place in March 2021. Preferred Reporting Items for Transparent Reporting of Systematic Reviews and Meta-Analysis guided articles identification and selection. The quality assessment followed Joanna Briggs International's critical appraisal tool. Results. We identified six articles as assessed the well-being of institutionalized aging people during the pandemic. The results suggest that infection prevention and control measures implemented in nursing homes may affect the well-being perceived by aging residents. They experience loss of human contact and the loss of space for circulation, with cognitive decline, loss of functional capacity, emotional condition, and loneliness. Conclusions. The infection prevention and control measures implemented in nursing homes in the COVID-19 pandemic appear to influence negatively residents' well-being

Keywords: Nursing Homes; Aging; Well-Being; COVID-19; Infection Control. 


\section{Introdução}

Os primeiros casos de Covid-19 surgiram na cidade de Wuhan, na China, no final do ano 2019. Desde então, o mundo assiste a uma pandemia que já afetou mais de 120 milhões de pessoas globalmente e causou 3 milhões de mortos (WHO, 2021). As pessoas idosas, pelas suas condições de saúde e a idade avançada, são o grupo de maior vulnerabilidade, com um risco acrescido de hospitalização e morte, por agravamento da doença (Yanez, Weiss, Romand \& Treggiari, 2020).

As Estruturas Residenciais para Pessoas Idosas (ERPI) são muito permeáveis à presença de surtos por COVID-19, pelo facto de integrar várias pessoas idosas, em que o distanciamento social nem sempre é conseguido, o que se traduz em elevadas taxas de ataque, quer nos residentes, quer nos funcionários (Blain et al., 2020; Vijh et al., 2021).

Para evitar a disseminação do vírus, as ERPI adotaram as medidas recomendadas pelas autoridades de saúde internacionais (WHO, 2020; ECDC, 2021), que incluem o cumprimento do distanciamento social e a implementação de medidas de isolamento, como a quarentena ou a privação de contacto físico dos residentes com os familiares. Estas medidas, por se prolongarem no tempo, podem trazer implicações importantes no bem-estar dos residentes.

O isolamento social, secundário à implementação das medidas de prevenção e controle de infeção por COVID-19, teve repercussões importantes na perceção da saúde física e mental da população geral (Ruiz et al., 2020), com níveis elevados de ansiedade e depressão e baixos níveis de saúde mental (Smith et al., 2020). Na população mais idosa, persiste a depressão e a ansiedade, com aterações da qualidade do sono, inatividade física (Sepúlveda-Loyola et al., 2020), sentimentos de solidão e perda de qualidade de vida (Kasar, K. S., \& Karaman, 2021). Nesta população, o isolamento social está associado a um aumento significativo da morbilidade e mortalidade (Roy, Jain, Golamari, Vunnam, \& Sahu, 2020). Desta forma, e considerando as repercurssões elencadas anteriormente, também o bem-estar ficará afetado.

O conceito de bem estar tem evoluído ao longo das últimas décadas sendo que é hoje utilizado de forma abrangente nas várias áreas científicas. Assim, podemos definir o bemestar como construto multifacetado, um processo intencional de crescimento individual, integração de experiências e conexão significativa com outras pessoas, refletindo objetivos e forças pessoalmente valorizadas e resultando no sentimento de estar bem e viver consoante os seus valores (McMahon \& Fleury, 2012; Mendes, 2020).

Não existem muito estudos que explorem a questão do bem-estar da pessoa idosa após o surgimento da pandemia e os mais divulgados são com idosos residentes na comunidade.

O nosso artigo integra um projeto mais amplo, eduCOVID: Mobile application for an integrated response to Covid-19 and beyond in nursing homes, e tem como objetivo identificar estudos que permitam compreender o bem-estar das pessoas idosas residentes em ERPI face ao cumprimento das medidas de prevenção e controle de infeção para a COVID-19.

\section{Metodologia}

E na população idosa institucionalizada? As medidas de prevenção e controle de infeção para a COVID-19 influenciaram o bem-estar das pessoas idosas residentes em ERPI?

Para dar resposta à questão de partida colocada, realizou-se uma revisão integrativa da literatura, seguindo as seguintes etapas: definição da pergunta de investigação, estabelecimento dos critérios de inclusão e exclusão, definição da informação a extrair dos estudos, avaliação dos estudos, interpretação dos resultados e síntese do conhecimento (Mendes, Silveira \& Galvão, 2008). 
A questão de investigação foi desenhada de acordo com a estratégia PCC (População, Conceito, Contexto) (Peters et al., 2020), sendo P - Pessoas idosas sujeitas a medidas de prevenção e controle de infeção para a COVID-19, C - Bem-estar, C - ERPI.

A pesquisa dos estudos foi conduzida no início do mês de março de 2021, através do motor de busca EBSCO, nas bases de dados Academic Search Complete, Medical Literature Analysis and Retrieval System Online (MEDLINE), Cumulative Index to Nursing and Allied Health Literature (CINAHL) e Cochrane Database of Systematic Reviews. Foi ainda realizada pesquisa na US National Library of Medicine (Pubmed), SCOPUS e Web of Science.

Foram definidos critérios de inclusão e de exclusão (Tabela 1).

Tabela 1. Critérios de elegibilidade dos estudos, 2021.

\begin{tabular}{|c|c|c|}
\hline & Critérios de Inclusão & Critérios de Exclusão \\
\hline Participantes & $\begin{array}{l}\text { Pessoas idosas institucionalizadas } \\
\text { ou seus familiares }\end{array}$ & $\begin{array}{l}\text { Crianças, grávidas, idosos } \\
\text { institucionalizados antes da } \\
\text { pandemia COVID-19 }\end{array}$ \\
\hline Conceito & Bem-estar, saúde & $\begin{array}{c}\text { Estudos sem uma abordagem } \\
\text { clara ao conceito de bem-estar } \\
\text { ou saúde }\end{array}$ \\
\hline Contexto & ERPI & Comunidade, Hospital \\
\hline Estudos & $\begin{array}{l}\text { Estudos primários (quantitativos e } \\
\text { qualitativos), disponíveis em texto } \\
\text { completo, publicados desde } 2019 \\
\text { até à atualidade, sem de idioma. }\end{array}$ & $\begin{array}{c}\text { Artigos de opinião, editoriais, } \\
\text { cartas ao editor ou artigos de } \\
\text { revisão. }\end{array}$ \\
\hline
\end{tabular}

A identificação e seleção dos estudos foi conduzida por dois investigadores, combinando com os operadores boleanos OR ou AND, os descritores Medical Subject Headings (Mesh) e palavras-chave, correspondentes a cada uma das dimensões identificadas em PCC. Assim, utilizou-se a seguinte estratégia de pesquisa: ((Residents OR aging OR ageing OR "Older persons" OR "older people" OR elderly OR aged) AND ((Isolation* OR quarantin* OR "Containment strategy" OR "Containment of Biohazards" OR Measur* OR Containment" OR "social distance") AND ("Disease Transmission" OR "Infectious Disease Transmission, Professional-to-Patient" OR "Infectious Disease Transmission, Patient-toProfessional" OR "Disease Transmission, Patient-to-Professional" OR "Disease Transmission, Professional-to- Patient" OR "Infection Control" OR "Risk Factors" OR "Risk reduction behaviour" OR "Harm reduction") AND ("COVID-19" OR "2019-nCoV" OR "SARS-COV2"))) AND (Well-being OR wellbeing OR health*) AND ("Residential Facilities" OR "Assisted Living Facilities" OR "Homes for the Aged" OR "Nursing Homes" OR "LongTerm Care" OR "residential aged care" OR "Long-Term Care Facilities").

Foram identificados 1051 artigos, 30 na Academic Search Complete, 38 na CINAHL, 1 na Cochrane Database of Systematic Reviews, 65 na MEDLINE, 80 na Pubmed, 805 na SCOPUS e 32 na Web of Science. Após a análise do título e do resumo selecionámos 26 artigos para análise do texto integral. Destes, excluímos 20, o que permitiu integrar 6 artigos na nossa amostra final. Estes foram sujeitos à avaliação da qualidade de acordo com a Joanna Briggs International (Lockwood et al, 2015), tendo-se verificado que cumprem os critérios, com avaliações cegas entre dois dos investigadores, a todos os artigos integrados na amostra afinal.

Todos os estudos foram classificados quanto ao nível de evidência: Nível I - meta-análise de ensaios clínicos randomizados (RCT); Nível II - RCT; Nível III - ensaios controlados sem randomização (quase-experimental); Nível IV - estudos de caso-controle ou coorte; Nível V - revisões sistemáticas de estudos descritivos e qualitativos (metassíntese); Nível VI - estudo descritivo ou qualitativo; Nível VII - opinião de autoridades ou relatórios de comissões de especialistas (Ackley, Swan, Ladwig, \& Tucker, 2008). 
A pesquisa e seleção de artigos foi suportada pelo fluxograma PRISMA recomendado pela Preferred Reporting Items for Transparent Reporting of Systematic Reviews and MetaAnalyses (Moher, Liberati, Tetzlaff, Altman, 2009) (Figura 1).

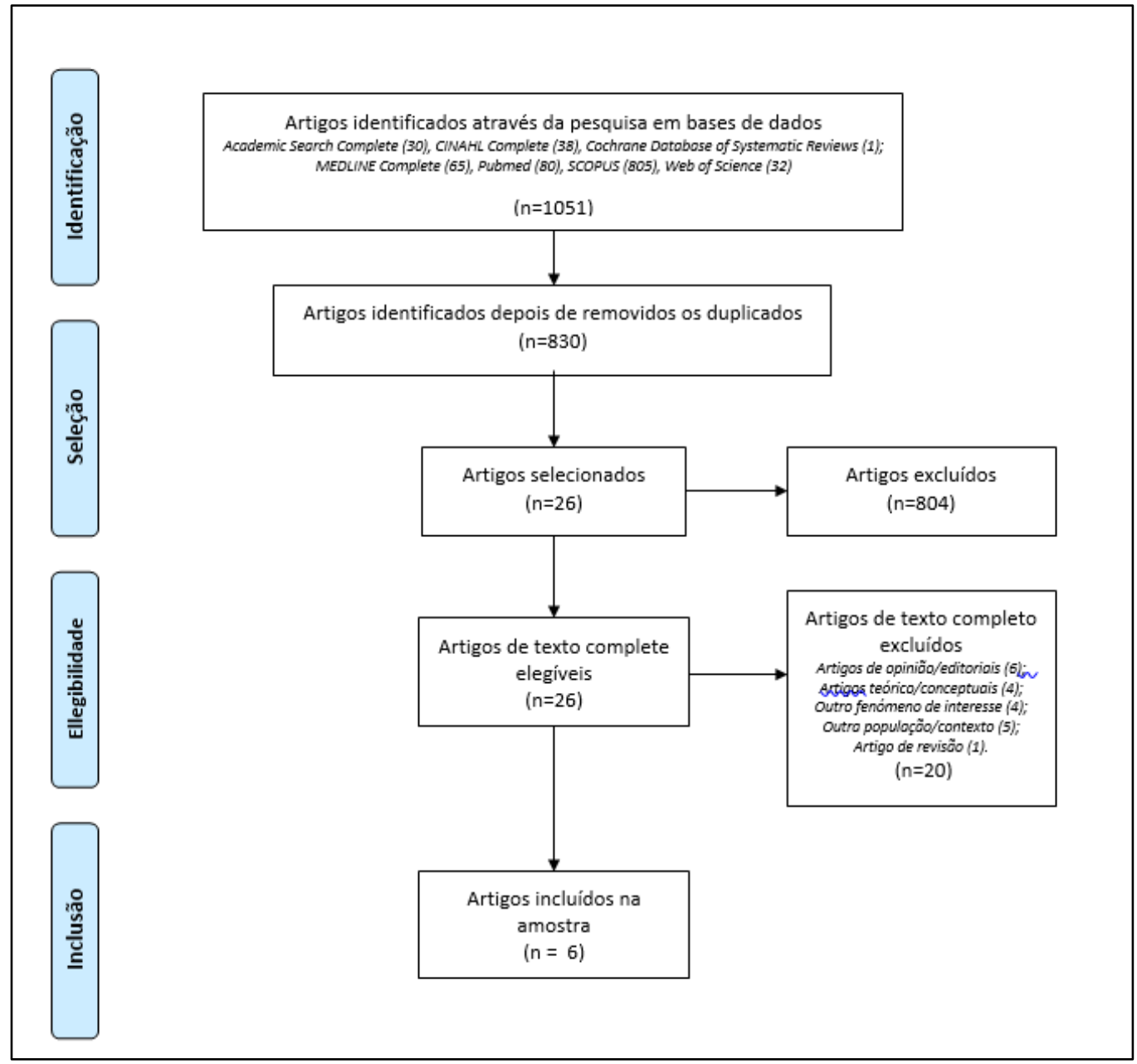

Fig. 1. Fluxograma do processo de seleção e identificação dos estudos para a revisão integrativa, 2021.

Os artigos incluídos na amostra final foram sujeitos a uma análise crítica e a uma síntese qualitativa. Por se tratar de uma revisão integrativa, a pesquisa não foi submetida a uma Comissão de Ética, no entanto os investigadores tiveram a preocupação de serem fiéis aos resultados utilizados nos estudos primários selecionados.

\section{Resultados}

Entre 2020 e 2021 identificámos seis artigos que avaliam o bem-estar em pessoas idosas institucionalizadas durante a pandemia. Os artigos que compõem a amostra final representam países dos continentes Asiático (1), Europeu (4) e Americano (1). Quatro dos estudos são quantitativo-descritivos, um estudo descritivo-misto e um qualitativo (fenomenológico) (Tabela 2). 
Tabela 2. Características dos estudos selecionados, 2021.

\begin{tabular}{|c|c|c|c|c|c|}
\hline $\begin{array}{l}\text { Autor/ } \\
\text { ano }\end{array}$ & $\begin{array}{l}\text { País/ } \\
\text { Participantes }\end{array}$ & $\begin{array}{c}\text { Desenho } \\
\text { do } \\
\text { estudo }\end{array}$ & Objetivo & Resultados & $\begin{array}{l}\text { Nível de } \\
\text { evidência }\end{array}$ \\
\hline $\begin{array}{l}\text { Chee, } \\
2020\end{array}$ & $\begin{array}{c}\text { Malásia } \\
10 \text { residentes }\end{array}$ & $\begin{array}{l}\text { Estudo } \\
\text { fenomeno } \\
\text { lógico } \\
\text { (Entrevist } \\
\text { as } \\
\text { individuai } \\
\text { s, em } \\
\text { profundid } \\
\text { ade) }\end{array}$ & $\begin{array}{l}\text { Explorar as } \\
\text { experiências } \\
\text { vividas por } \\
\text { idosos em } \\
\text { ERPI durante a } \\
\text { pandemia } \\
\text { COVID-19, } \\
\text { especialmente } \\
\text { sua perspetiva } \\
\text { sobre como } \\
\text { isso afetou a } \\
\text { sua rotina } \\
\text { diária, } \\
\text { relacionamento } \\
\text { s e bem-estar } \\
\text { geral. }\end{array}$ & $\begin{array}{l}\text { Os participantes } \\
\text { sentem-se } \\
\text { desconectados } \\
\text { com o mundo que } \\
\text { "encolheu". } \\
\text { Verbalizam } \\
\text { sentimentos de } \\
\text { medo e incerteza. } \\
\text { A restrição da } \\
\text { interação com os } \\
\text { outros leva ao } \\
\text { isolamento social, } \\
\text { solidão e } \\
\text { frustração. } \\
\text { As medidas de } \\
\text { confinamento } \\
\text { levaram à redução } \\
\text { da mobilidade e à } \\
\text { perda de } \\
\text { capacidade de } \\
\text { movimento. } \\
\text { Conduziram a } \\
\text { distúrbios do sono, } \\
\text { causado pelas } \\
\text { sestas diurnas } \\
\text { mais frequentes. } \\
\text { Impediram os } \\
\text { residentes de } \\
\text { encontrar um } \\
\text { espaço para estar } \\
\text { só, longe dos } \\
\text { outros residentes. } \\
\text { A vida é vivida } \\
\text { como uma } \\
\text { experiência de } \\
\text { sofrimento. }\end{array}$ & VI \\
\hline $\begin{array}{l}\text { El Haj } \\
\text { et al., } \\
2020\end{array}$ & $\begin{array}{c}\text { França } \\
58 \text { residentes } \\
\text { com doença } \\
\text { de Alzheimer }\end{array}$ & $\begin{array}{c}\text { Estudo } \\
\text { descritivo } \\
\text { (Hospital } \\
\text { Anxiety } \\
\text { and } \\
\text { Depressio } \\
\text { n Scale) }\end{array}$ & $\begin{array}{l}\text { Investigar os } \\
\text { efeitos das } \\
\text { medidas contra } \\
\text { Covid-19 na } \\
\text { saúde mental } \\
\text { de participantes } \\
\text { com doença de } \\
\text { Alzheimer (DA) } \\
\text { que vivem em } \\
\text { lares de idosos } \\
\text { na França. }\end{array}$ & $\begin{array}{l}\text { Os participantes } \\
\text { relataram maior } \\
\text { depressão }(p= \\
0,005) \text { e } \\
\text { ansiedade }(\mathrm{p}= \\
0,004) \text { durante o } \\
\text { confinamento do } \\
\text { que antes da } \\
\text { pandemia por } \\
\text { Covid-19. }\end{array}$ & VI \\
\hline
\end{tabular}




\begin{tabular}{|c|c|c|c|c|c|}
\hline $\begin{array}{l}\text { Autor/ } \\
\text { ano }\end{array}$ & $\begin{array}{c}\text { País/ } \\
\text { Participantes }\end{array}$ & $\begin{array}{c}\text { Desenho } \\
\text { do } \\
\text { estudo }\end{array}$ & Objetivo & Resultados & $\begin{array}{l}\text { Nível de } \\
\text { evidência }\end{array}$ \\
\hline $\begin{array}{l}\text { Lomb } \\
\text { ardo } \\
\text { et al., } \\
2020\end{array}$ & $\begin{array}{c}\text { Itália } \\
1356 \text { ERPI } \\
(100806 \\
\text { residentes })\end{array}$ & $\begin{array}{c}\text { Estudo } \\
\text { descritivo } \\
\text { (Question } \\
\text { ário) }\end{array}$ & $\begin{array}{c}\text { Documentar a } \\
\text { frequência de } \\
\text { eventos } \\
\text { adversos que } \\
\text { ocorreram em } \\
\text { ERPI italianas } \\
\text { durante os } \\
\text { meses em que } \\
\text { o vírus teve sua } \\
\text { maior taxa de } \\
\text { propagação (do } \\
\text { início de } \\
\text { fevereiro ao } \\
\text { início de maio); } \\
\text { e identificar os } \\
\text { determinantes } \\
\text { e atributos } \\
\text { associados à } \\
\text { ocorrência de } \\
\text { eventos } \\
\text { adversos } \\
\text { durante a } \\
\text { pandemia. }\end{array}$ & $\begin{array}{l} \\
\text { Durante a } \\
\text { pandemia, as } \\
\text { ERPI que } \\
\text { relataram efeitos } \\
\text { adversos também } \\
\text { relataram maior } \\
\text { frequência de uso } \\
\text { de psicofármacos } \\
\text { e contenção física } \\
\text { quando } \\
\text { comparados } \\
\text { àqueles que não } \\
\text { relataram } \\
\text { quaisquer efeitos } \\
\text { adversos. }\end{array}$ & VI \\
\hline $\begin{array}{l}\text { McArt } \\
\text { hur et } \\
\text { al., } \\
2021\end{array}$ & $\begin{array}{c}\text { Canadá } \\
765 \\
\text { residentes (7 } \\
\text { ERPI) }\end{array}$ & $\begin{array}{c}\text { Estudo } \\
\text { descritivo } \\
\text { (InterRAI } \\
\text { LTCF, } \\
\text { standardiz } \\
\text { ed } \\
\text { assessme } \\
\text { nt tool) }\end{array}$ & $\begin{array}{c}\text { Avaliar o efeito } \\
\text { das estratégias } \\
\text { usadas para } \\
\text { mitigar o } \\
\text { agravamento } \\
\text { dos resultados } \\
\text { de saúde } \\
\text { mental durante } \\
\text { a pandemia de } \\
\text { COVID-19. }\end{array}$ & $\begin{array}{c}\text { Durante o } \\
\text { confinamento os } \\
\text { residentes tinham } \\
\text { pior mobilidade } \\
\text { funcional, } \\
\text { instabilidade de } \\
\text { saúde e } \\
\text { comprometimento } \\
\text { cognitivo. } \\
\text { Experimentavam } \\
\text { problemas } \\
\text { comportamentais } \\
\text { e as mulheres } \\
\text { eram mais } \\
\text { propensas a ter } \\
\text { sintomas } \\
\text { depressivos. } \\
\text { Em residentes } \\
\text { com pior } \\
\text { comprometimento } \\
\text { cognitivo o } \\
\text { delirium foi mais } \\
\text { frequente, e } \\
\text { menos frequente } \\
\text { se apresentassem } \\
\text { níveis mais altos } \\
\text { de envolvimento } \\
\text { social. Os } \\
\text { residentes com } \\
\text { demência ou } \\
\text { deficiência } \\
\text { cognitiva eram } \\
\text { mais propensos a } \\
\text { ter problemas } \\
\text { comportamentais. }\end{array}$ & VI \\
\hline
\end{tabular}




\begin{tabular}{|c|c|c|c|c|c|}
\hline $\begin{array}{l}\text { Autor/ } \\
\text { ano }\end{array}$ & $\begin{array}{c}\text { País/ } \\
\text { Participantes }\end{array}$ & $\begin{array}{l}\text { Desenho } \\
\text { do } \\
\text { estudo }\end{array}$ & Objetivo & Resultados & $\begin{array}{l}\text { Nível de } \\
\text { evidência }\end{array}$ \\
\hline $\begin{array}{l}\text { O'Cao } \\
\text { imh et } \\
\text { al., } \\
2020\end{array}$ & $\begin{array}{c}\text { Irlanda } \\
225 \text { familiares } \\
\text { de residentes }\end{array}$ & $\begin{array}{c}\text { Estudo } \\
\text { descritivo } \\
\text { (Subjectiv } \\
\text { e } \\
\text { psycholog } \\
\text { ical well- } \\
\text { being: } \\
\text { World } \\
\text { Health } \\
\text { Organizati } \\
\text { on Five } \\
\text { Well- } \\
\text { being } \\
\text { Index } \\
\text { (WHO-5); } \\
\text { Lonelines } \\
\text { s: } \\
\text { University } \\
\text { of } \\
\text { California, } \\
\text { Los } \\
\text { Angeles } \\
\text { (UCLA)). }\end{array}$ & $\begin{array}{l}\text { Examinar o } \\
\text { efeito das } \\
\text { restrições de } \\
\text { visitas sobre a } \\
\text { perceção de } \\
\text { solidão, bem- } \\
\text { estar e } \\
\text { qualidade de } \\
\text { vida entre } \\
\text { visitantes de } \\
\text { residentes com } \\
\text { e sem } \\
\text { comprometime } \\
\text { nto cognitivo } \\
\text { (Cl) em ERPI } \\
\text { irlandesas. }\end{array}$ & $\begin{array}{c}\text { De acordo com a } \\
\text { perceção dos } \\
\text { familiares cerca de } \\
80 \% \text { dos } \\
\text { residentes tiveram } \\
\text { declínio cognitivo } \\
\text { durante a } \\
\text { aplicação das } \\
\text { restrições para } \\
\text { controle da } \\
\text { COVID-19. } \\
\text { Relatam } \\
\text { mudanças no } \\
\text { humor, atividades } \\
\text { da vida diária ou } \\
\text { cognição, } \\
\text { enquanto } \\
\text { participavam em } \\
\text { telefonemas ou } \\
\text { outras interações. }\end{array}$ & VI \\
\hline $\begin{array}{l}\text { Verbe } \\
\text { ek ey } \\
\text { al., } \\
2020\end{array}$ & $\begin{array}{c}\text { Holanda } \\
30 \text { familiares } \\
\text { de residentes } \\
\text { (26 ERPI) }\end{array}$ & $\begin{array}{l}\text { Estudo } \\
\text { misto } \\
\text { (Question } \\
\text { ário e } \\
\text { entrevista) }\end{array}$ & $\begin{array}{l}\text { Descrever a } \\
\text { adesão às } \\
\text { práticas de } \\
\text { prevenção e } \\
\text { controle de } \\
\text { infeção e o seu } \\
\text { impacto no } \\
\text { bem-estar dos } \\
\text { residentes, } \\
\text { seus } \\
\text { cuidadores } \\
\text { familiares e } \\
\text { funcionários. }\end{array}$ & $\begin{array}{l}\text { O regresso das } \\
\text { visitas aos lares } \\
\text { durante a } \\
\text { pandemia por } \\
\text { COVID-19 foi } \\
\text { percebido pelos } \\
\text { familiares como } \\
\text { um momento que } \\
\text { trouxe bem-estar } \\
\text { ao residente. A } \\
\text { impossibilidade do } \\
\text { contacto físico foi } \\
\text { relatada como } \\
\text { muito difícil, } \\
\text { principalmente } \\
\text { para os residentes } \\
\text { com demência. } \\
\text { Em alguns casos, } \\
\text { foi relatado que os } \\
\text { residentes não } \\
\text { reconheceram } \\
\text { suas famílias } \\
\text { devido ao longo } \\
\text { período de } \\
\text { restrições de } \\
\text { visitas. }\end{array}$ & VI \\
\hline
\end{tabular}

No global, os estudos revelam que os residentes de ERPI, no decurso da aplicação das medidas de prevenção e controle da infeção por COVID-19, experienciaram declínio cognitivo, perda de capacidade funcional, mal-estar emocional e solidão. Estas experiências resultam da perda do contacto humano e da perda de espaço de circulação. A primeira decorre do isolamento provocado pela limitação das visitas de familiares e limitação dos contactos com os profissionais, pelo uso de equipamento de proteção individual. A segunda decorre da impossibilidade de saída para o exterior da instituição, do confinamento nos quartos, ou da mudança de quarto, pela necessidade de segregação. 


\section{Discussão}

Os resultados da amostra bibliográfica apontam para um impacto negativo das medidas de restrição e controle da pandemia na funcionalidade da pessoa idosa, associada à perda de capacidade física pela diminuição da mobilidade e dificuldades acrescidas na marcha (Chee, 2020; MacArtur et al, 2021), depressão (El Hag et al., 2020), maior uso de psicofármacos (Lombardo et al., 2020), declínio cognitivo (O'Caoimh et al., 2020), entre outros. Estes achados são preocupantes e preditores do aparecimento de co-morbilidades e complicações associadas à síndrome de imobilidade.

Por outro lado, as alterações ambientais, a mudança de quarto e as modificações no circuito de acessibilidades aos diferentes espaços dentro das instituições poderão contribuir para o aumento das quedas e dos episódios de confusão aguda. Recorde-se que a prevalência de quedas é elevada nos primeiros 5 dias após a institucionalização, o que é justificado pelo facto de os idosos não conhecerem o espaço físico e pela presença de pessoas estranhas ao seu ambiente, como os funcionários e os outros idosos (Baixinho \& Dixe, 2017; Oliveira, Baixinho, \& Henriques, 2018).

O aumento da prescrição de psicofármacos na pessoa com demência e, em particular de antipsicóticos, sugerido pelo trabalho de Lombardo et al. (2020), suscita preocupação. Os antipsicóticos, prescritos para comportamentos e sintomas psicológicos da demência, estão associados a aumento da morbilidade e mortabilidade (Corbett, Burns \& Ballard, 2014). Regra geral, considera-se que nesta população os riscos superam os benefícios, devendo a sua utilização ser restrita aos casos de falência de medidas não farmacológicas e quando os sintomas causam sofrimento significativo e/ou acarretam risco de dano para a pessoa ou para terceiros. O facto da maioria dos antipsicóticos ser prescrito "off-label", sem indicação terapêutica aprovada para os sintomas psicológicos da demência, o comprometimento da compreensão e autonomia destas pessoas para prestar um consentimento informado, esclarecido e livre, e a potencial falta de envolvimento dos seus representantes na decisão de utilizar antipsicóticos, amplificam esta problemática.

Os planos de contingência desta revisão revelam uma visão 'infetocentrica', não prevendo a avaliação da efetividade das medidas implementadas sobre as síndromes geriátricas associadas ao processo de envelhecimento. Em paralelo, os mesmos estudos não garantem a possibilidade de participação dos idosos institucionalizados na tomada de decisão sobre as referidas medidas, na organização de atividades de promoção da mobilidade e participação social na vida da instituição, aspetos centrais para o fomento da atividade física e preservação da autonomia e qualidade de vida. Maia e colaboradores (2020) defendem que é crucial que as pessoas idosas se mantenham com autonomia e independência funcional, evidenciando capacidade de assegurar a gestão das suas atividades de lazer, de convívio social e trabalho, independentemente da presença ou não de comorbilidades. Esta ideia é reforçada por Beard et al. (2016), ao advogarem que a interação da pessoa idosa com o seu ambiente, suportado na sua autonomia e independência, devem constituir-se como o alicerce para a abordagem de saúde ao idoso.

Entendendo o conceito de mal-estar emocional como o desconforto ímpar, estado emocional vivenciado por um indivíduo em resposta a um stressor específico, ou resposta que resulta em dano, seja temporário ou permanente, para a pessoa (Ridner, 2004), os resultados obtidos são fortemente sugestivos da sua ocorrência na pessoa idosa residente em ERPI. Este mal-estar emocional é manifestado pelos residentes em ERPI por uma sensação de desligamento, desconexão com um mundo que se tornou mais pequeno que "encolheu", que Ihes provoca isolamento social, solidão e, paradoxalmente, Ihes dificulta o encontrar um espaço onde consigam estar sós, longe dos restantes residentes (Chee, 2020).

São também relatadas mudanças de humor durante atividades de interação (O'Caoimh et al., 2020), sentimentos de medo e incerteza, frustração, sofrimento (Chee, 2020), maiores níveis de depressão (Lombardo et al., 2020) e ansiedade do que antes do confinamento imposto pela pandemia por COVID-19 (El Hag et al., 2020; MacArtur et al., 2021). 
É importante considerar que estes achados poderem ser preditores de instalação de quadros de perturbação mental e declínio cognitivo, necessitando que sejam introduzidas medidas de controle e prevenção do impacto psicológico e emocional da COVID-19 na pessoa idosa (Mukhtar, 2020). Se as medidas implementadas durante o confinamento têm um contributo positivo na prevenção e controle da doença, o mal-estar emocional nos idosos requer uma atenção particular pois estes integram o grupo que tem experienciado o isolamento social mais prolongado (Lee et al., 2020). Algumas ERPI implementaram medidas para contrariar este isolamento dos residentes, promovendo videoconferências ou visitas à janela (McArthur et al., 2021).

Os seis estudos incluídos apresentam um baixo nível de evidência, o que pode ser justificado pela dificuldade em conduzir ensaios aleatorizados e controlados nestas circunstâncias. Acresce que três dos seis estudos reportam perceções dos familiares ou das ERPI, e não dos residentes. Outra limitação diz respeito ao estudo de Lombardo et al. (2020), que se baseia no auto-reporte. É, portanto, fundamental prosseguir a investigação no campo do bem-estar da pessoa idosa institucionalizada.

\section{Conclusões}

A evidência incluída nesta revisão integrativa sugere que as medidas de prevenção e controle de infeção para a COVID-19 em ERPI condicionaram a perda de contacto humano e a perda de espaço de circulação e influenciaram negativamente o bem-estar das pessoas idosas, estando associadas a efeitos como redução da funcionalidade, perda de capacidade física, depressão, ansiedade, declínio cognitivo e solidão. Recomenda-se a realização de estudos com desenho mais robusto, nomeadamente que permitam investigar a associação entre a restrição de atividade e o aumento de complicações de saúde e acidentes nesta população.

Não obstante a necessidade de controlar a pandemia numa perspetiva epidemiológica, importa monitorizar também o bem-estar das pessoas idosas institucionalizadas e o seu contexto, garantindo a implementação de medidas que se coadunem com as suas necessidades e minorem os efeitos negativos do isolamento.

\section{Referências}

Ackley, B. J., Swan, B. A., Ladwig, G., \& Tucker, S. (2008). Evidence-based nursing care guielines: Medical-surgical interventions. St. Louis, MO: Mosby Elsevier.

Baixinho, C.L., \& Dixe, M.A. (2017). Cuáles son las prácticas y comportamientos de los mayores institucionalizados para prevenir las caídas? Rev Index de Enfermaria, 26(4), 255-59.

Beard, J. R., Officer, A., de Carvalho, I. A., Sadana, R., Pot, A. M., Michel, J. P., Lloyd-Sherlock, P., Epping-Jordan, J. E., Peeters, G., Mahanani, W. R., Thiyagarajan, J. A., \& Chatterji, S. (2016). The World report on ageing and health: a policy framework for healthy ageing. Lancet, 387(10033), 2145-2154.

Blain, H., Rolland, Y., Tuaillon, E., Giacosa, N., Albrand, M., Jaussent, A., ... \& Bousquet, J. (2020). Efficacy of a test-retest strategy in residents and health care personnel of a nursing home facing a COVID-19 outbreak. Journal of the American Medical Directors Association, 21(7), 933-936.

Chee, S. Y. (2020). COVID-19 Pandemic: The Lived Experiences of Older Adults in Aged Care Homes. Millennial Asia, 11(3), 299-317.

Corbett, A., Burns, A., \& Ballard, C. (2014). Don't use antipsychotics routinely to treat agitation and aggression in people with dementia. Bmj, 349.

European Centre for Disease Prevention and Control. (2020). Infection prevention and control and preparedness for COVID-19 in healthcare settings. In: https://www.ecdc.europa.eu/en/publications-data/infection-prevention-and-control-andpreparedness-covid-19-healthcare-settings 
El Haj, M., Altintas, E., Chapelet, G., Kapogiannis, D., \& Gallouj, K. (2020). High depression and anxiety in people with Alzheimer's disease living in retirement homes during the covid-19 crisis. Psychiatry research, 291, 113294.

Kasar, K. S., \& Karaman, E. (2021). Life in lockdown: Social Isolation, Loneliness and Quality of Life in the Elderly During the COVID-19 Pandemic: A Scoping Review. Geriatric Nursing.

Lee, K., Jeong, G. C., \& Yim, J. (2020). Consideration of the Psychological and Mental Health of the Elderly during COVID-19: A Theoretical Review. International journal of environmental research and public health, 17(21), 8098.

Lockwood, C., Munn, Z., \& Porritt, K. (2015). Qualitative research synthesis: methodological guidance for systematic reviewers utilizing meta-aggregation. International Journal of Evidence-Based Healthcare, 13(3), 179-187.

Lombardo, F. L., Salvi, E., Lacorte, E., Piscopo, P., Mayer, F., Ancidoni, A., ... \& Nursing Home Study Group. (2020). Adverse events in Italian nursing homes during the COVID-19 epidemic: a national survey. Frontiers in psychiatry, 11.

Maia, L., Colares, T., Moraes, E., Costa, S., \& Caldeira, A. (2020). Idosos robustos na atenção primária: fatores associados ao envelhecimento bem-sucedido. Revista de Saúde Pública, 54, 35

Mendes, K., Silveira, R., \& Galvão, C. (2008). Revisão integrativa: método de pesquisa para a incorporação de evidências na saúde e na enfermagem. Texto \& contexto-enfermagem, 17(4), 758-764.

Mendes, J. (2020). Envelhecimento (s), qualidade de vida e bem-estar. A Psicologia em suas Diversas Áreas de Atuação, 132-144.

McArthur, C., Saari, M., Heckman, G. A., Wellens, N., Weir, J., Hebert, P., ... \& Hirdes, J. P. (2021). Evaluating the effect of COVID-19 pandemic lockdown on long-term care residents' mental health: a data-driven approach in new brunswick. Journal of the American Medical Directors Association, 22(1), 187-192.

McMahon, S., \& Fleury, J. (2012). Wellness in Older Adults: A Concept Analysis. Nursing Forum, 47(1), 39-51. In: https://doi.org/https://doi.org/10.1111/j.1744-6198.2011.00254.x

Moher D, Liberati A, Tetzlaff J, Altman DG. (2009). The PRISMA Group (2009). Preferred Reporting Items for Systematic Reviews and Meta-Analyses: the PRISMA Statement. PLoS Med,6(7):e1000097. doi: 10.1371/journal.pmed.1000097

Mukhtar S. (2020). Psychological impact of COVID-19 on older adults. Current medicine research and practice, 10(4), 201-202.

O'Caoimh, R., O'Donovan, M. R., Monahan, M. P., Dalton O'Connor, C., Buckley, C., Kilty, C., ... \& Cornally, N. (2020). Psychosocial Impact of COVID-19 Nursing Home Restrictions on Visitors of Residents With Cognitive Impairment: A Cross-Sectional Study as Part of the Engaging Remotely in Care (ERiC) Project. Frontiers in psychiatry, 11, 1115.

Oliveira, T., Baixinho, C.L., \& Henriques, M, ㄹ A. (2018). Multidimensional risk of falls in elderly. Revista Brasileira em Promoção da Saúde. , 31(2): 1-9.

Peters MDJ, Godfrey C, Mclnerney P, Munn Z, Tricco AC, Khalil, H. (2020). Chapter 11: Scoping Reviews (2020 version). In: Aromataris E, Munn Z (Editors). JBI Manual for Evidence Synthesis. Available from https://synthesismanual.jbi.global https://doi.org/10.46658/JBIMES-20-12

Ridner SH. (2004). Psychological distress: concept analysis. J Adv Nurs, 45(5):536-45. doi: 10.1046/j.1365-2648.2003.02938.x.

Roy, J., Jain, R., Golamari, R., Vunnam, R., \& Sahu, N. (2020). COVID-19 in the geriatric population. International journal of geriatric psychiatry, 35(12), 1437-1441.

Ruiz, M. C., Devonport, T. J., Chen-Wilson, C. H. J., Nicholls, W., Cagas, J. Y., FernandezMontalvo, J., ... \& Robazza, C. (2020). A Cross-Cultural Exploratory Study of Health Behaviors and Wellbeing During COVID-19. Frontiers in psychology, 11. 
Smith, L., Jacob, L., Yakkundi, A., McDermott, D., Armstrong, N. C., Barnett, Y., ... \& Tully, M. A. (2020). Correlates of symptoms of anxiety and depression and mental wellbeing associated with COVID-19: a cross-sectional study of UK-based respondents. Psychiatry research, 291, 113138.

Sepúlveda-Loyola, W., Rodríguez-Sánchez, I., Pérez-Rodríguez, P., Ganz, F., Torralba, R., Oliveira, D. V., \& Rodríguez-Mañas, L. (2020). Impact of social isolation due to COVID-19 on health in older people: Mental and physical effects and recommendations. The journal of nutrition, health \& aging, 1-10.

Verbeek, H., Gerritsen, D. L., Backhaus, R., de Boer, B. S., Koopmans, R. T., \& Hamers, J. P. (2020). Allowing visitors back in the nursing home during the COVID-19 crisis: A Dutch national study into first experiences and impact on well-being. Journal of the American Medical Directors Association, 21(7), 900-904.

Vijh, R., Prairie, J., Otterstatter, M. C., Hu, Y., Hayden, A. S., Yau, B., ... \& Schwandt, M. (2021). Evaluation of a multisectoral intervention to mitigate the risk of severe acute respiratory coronavirus virus 2 (SARS-CoV-2) transmission in long-term care facilities. Infection Control and Hospital Epidemiology, 1.

World Health Organization. (2020). Infection prevention and control guidance for long-term care facilities in the context of COVID-19: interim guidance, 21 March 2020 (No. WHO/2019nCoV/IPC_long_term_care/2020.1). World Health Organization.

World Health Organization. (2021). WHO Coronavirus (COVID-19) Dashboard. World Health Organization. Available on: https://covid19.who.int/

Yanez, N. D., Weiss, N. S., Romand, J. A., \& Treggiari, M. M. (2020). COVID-19 mortality risk for older men and women. BMC Public Health, 20(1), 1-7. 